\title{
Convective drying kinetics of osmotically pretreated papaya cubes
}

\author{
João P. de L. Ferreira ${ }^{1}$, Deise S. de Castro ${ }^{1}$, Inacia dos S. Moreira ${ }^{1}$, Wilton P. da Silva ${ }^{1}$, \\ Rossana M. F. de Figueirêdo ${ }^{1}$ \& Alexandre J. de M. Queiroz ${ }^{1}$
}

\begin{abstract}
${ }^{1}$ Universidade Federal de Campina Grande/Centro de Tecnologia e Recursos Naturais/Programa de Pós-Graduação em Engenharia Agrícola. Campina Grande, PB, Brasil. E-mail: joaop_1@hotmail.com (Corresponding author) - ORCID: 0000-0003-1172-7259; deise_castro01@hotmail.com ORCID: 0000-0001-5040 -3295; inaciamoreira@ymail.com - ORCID: 0000-0002-7855-1895; wiltonps@uol.com.br - ORCID: 0000-0001-5841-6023; rossanamff@gmail.com - ORCID: 0000-0002-6187-5826 ; alexandrejmq@gmail.com - ORCID: 0000-0002-6880-5951
\end{abstract}

\begin{abstract}
This study assessed the fitting of mathematical models to the convective drying kinetics of osmotically pre-dehydrated papaya cubes. Papaya cubes were subjected to osmotic dehydration in sucrose solutions at 40 and $50^{\circ} \mathrm{Brix}$, at temperatures of 50 and $60^{\circ} \mathrm{C}$, followed by complementary convective drying in forced air circulation oven under three temperatures $\left(50,60\right.$ and $\left.70{ }^{\circ} \mathrm{C}\right)$ and constant air velocity of 1.0 $\mathrm{m} \mathrm{s}^{-1}$. Ten thin-layer drying mathematical models were fitted to the experimental data. The increase in air temperature and the decrease in osmotic solution concentration resulted in increased water removal rate. Based on the statistical indices, the Two Terms model was the one that best described the drying kinetics of the samples for all evaluated conditions. The effective diffusion coefficients increased with the elevation of air temperature, ranging from $1.766 \times 10^{-10}$ to $3.910 \times 10^{-6} \mathrm{~m}^{2} \mathrm{~s}^{-1}$, whereas the convective mass transfer coefficients ranged from $3.910 \times 10^{-7}$ to $1.201 \times 10^{-6} \mathrm{~m} \mathrm{~s}^{-1}$ with Biot number from 0.001 to 12.500 .
\end{abstract}

Key words: Carica papaya, osmotic dehydration, effective diffusivity

\section{Cinética de secagem convectiva de cubos de mamão pré-tratados osmoticamente}

RESUMO: Este estudo avaliou o ajuste de modelos matemáticos na cinética de secagem convectiva de cubos de mamão pré-desidratados osmoticamente. Os cubos de mamão foram submetidos à desidratação osmótica em soluções de sacarose a 40 e $50{ }^{\circ}$ Brix, em temperaturas de 50 e $60{ }^{\circ} \mathrm{C}$, seguida de secagem convectiva complementar em estufa com circulação forçada de ar sob três temperaturas $\left(50,60\right.$ e $\left.70{ }^{\circ} \mathrm{C}\right)$ e velocidade do ar constante de $1,0 \mathrm{~m} \mathrm{~s}^{-1}$. Aos dados experimentais foram ajustados dez modelos matemáticos de secagem em camada fina. O aumento da temperatura do ar e a diminuição da concentração da solução osmótica resultou em aumento da taxa de remoção de água. Com base nos índices estatísticos, o modelo de Dois Termos foi o que melhor descreveu a cinética de secagem das amostras para todas as condições avaliadas. Os coeficientes de difusão efetivos aumentaram com a elevação da temperatura do ar, variando de $1,766 \times 10^{-10}$ a 3,910 x $10^{-6}$ $\mathrm{m}^{2} \mathrm{~s}^{-1}$, enquanto os coeficientes convectivos de transferência de massa variaram entre 3,910 x $10^{-7}$ a $1,201 \mathrm{x}$ $10^{-6} \mathrm{~m} \mathrm{~s}^{-1}$ com número de Biot de 0,001 a 12,500.

Palavras-chave: Carica papaya, desidratação osmótica, difusividade efetiva 


\section{INTRODUCTION}

Brazil is the second largest producer of papaya in the world, having produced a total of 1.42 million tons in 2016 (FAOSTAT, 2018). Papaya has a fast ripening, which manifests itself immediately as a structural softening that, associated with its high moisture content and water activity, makes the product highly perishable, resulting in post-harvest losses throughout its chain (Kandasamy et al., 2012).

Convective drying, for its simplicity and low cost, compared to other drying methods such as lyophilization, is one of the most used technologies for the conservation of agricultural products. However, this method causes alterations in sensory and nutritional properties and in the bioactive compounds of the dry products (Gava et al., 2008; Orikasa et al., 2014). Such alterations can be minimized using combined drying methods, as proposed in the osmo-convective drying (Prosapio \& Norton, 2017; Dermesonlouoglou et al., 2018).

Mathematical modeling of the drying process is fundamental for understanding and providing information about the behavior of certain parameters that describe heat and mass transfer mechanisms (Silva et al., 2014a; Tzempelikos et al., 2015; Pacheco-Angulo et al., 2016), which can provide a solid basis for optimizing the process.

The quality of fit of the models to the experimental data can be assessed with different statistical indices; however, according to Kucuk et al. (2014), the best model to describe the drying curve of the product is the one with highest values of correlation coefficient, coefficient of determination, modeling efficiency and/or adjusted $\mathrm{R}^{2}$ and the lowest values of chisquare, mean squared deviation, relative mean percentage error, mean polarization error, standard error of estimation, residual sum of squares, reduced sum of squared errors and/ or residuals.

In this context, the objective was to assess the mathematical modeling of convective drying kinetics, at temperatures of 50,60 and $70^{\circ} \mathrm{C}$, of papaya cubes osmotically pre-dehydrated in sucrose solutions and to obtain the effective diffusivity coefficients and convective mass transfer coefficients.

\section{Material and Methods}

To conduct this study, the raw material used was ripe papaya fruits (Carica papaya L.) cv. Formosa, 2017 Season, purchased at the local market of the city of Campina Grande, PB, Brazil. Papaya fruits were washed with neutral detergent and subsequently sanitized with sodium hypochlorite solution (100 ppm) for $15 \mathrm{~min}$. The peel was removed with a stainlesssteel knife, and the seeds were discarded. The pulp was cut into cubes with dimensions of $20 \mathrm{~mm}$, measured with digital caliper (Absolute model, Mitutoyo, Brazil) with resolution of $0.01 \mathrm{~mm}$. The cubes were osmotically pre-dehydrated in sucrose solution (syrup) with 40 and $50^{\circ} \mathrm{Brix}$, in a cubes:syrup proportion of $1: 6$ (g:g), at temperatures of 50 and $60^{\circ} \mathrm{C}$. The osmotic dehydration (OD) process was carried out in a BOD chamber and lasted 4 $\mathrm{h}$, considering the maximum rate of water removal from the papaya cubes during the OD. The cubes were removed from the sucrose solution with plastic sieves and left on the bench to drain excess solution from the surface.

About $25 \mathrm{~g}$ of the osmotically dehydrated cubes were arranged, in a single layer, in stainless-steel rectangular baskets $(15 \times 12 \mathrm{~cm})$ and dried, in triplicate, in a forced air circulation oven (320/5 model, Foneman, Brazil) at temperatures of 50, 60, $70{ }^{\circ} \mathrm{C}$ and air velocity of $1.0 \mathrm{~m} \mathrm{~s}^{-1}$, determined by means of a digital anemometer (ITTHAL-300 model, Instrutemp, Brazil). Water loss was monitored by weighing on an electronic scale (AS5500C model, Marte, Brazil) with a resolution of $\pm 0.01 \mathrm{~g}$, at regular times of 5, 10, 20, 30 and $60 \mathrm{~min}$, until the samples reached constant mass. The data of drying kinetics were used to calculate the drying rates (Eq. 1) (Özdemira et al., 2017) and moisture content ratios (Eq. 2) (Galaz et al., 2017).

$$
\mathrm{DR}=\frac{\mathrm{M}_{\mathrm{t}_{0}}-\mathrm{M}_{\mathrm{t}_{0}+\Delta \mathrm{t}}}{\Delta \mathrm{t}}
$$

where:

DR - drying rate, $\mathrm{kg} \mathrm{kg}^{-1} \mathrm{~h}^{-1}$;

$\mathrm{M}_{\mathrm{t} 0}$ - moisture content at previous time, $\mathrm{kg} \mathrm{kg}^{-1} \mathrm{~d}$.b.;

$\mathrm{M}_{\mathrm{t} 0+\Delta \mathrm{t}}$ - moisture content at current time, $\mathrm{kg} \mathrm{kg}^{-1}$ d.b.; and,

$\Delta \mathrm{t}$ - difference between the current time $\left(\mathrm{t}_{\mathrm{i}}\right)$ and previous time $\left(\mathrm{t}_{0}\right)$ of drying, min.

$$
\mathrm{MR}=\frac{\mathrm{M}-\mathrm{M}_{\mathrm{e}}}{\mathrm{M}_{\mathrm{i}}-\mathrm{M}_{\mathrm{e}}}
$$

where:

MR - moisture content ratio, dimensionless;

M - moisture content at a specific time, d.b.;

$M_{e}$ - equilibrium moisture content, d.b.; and,

$M_{i} \quad$ - initial moisture content, d.b.

Different mathematical models were fitted to the experimental data of drying kinetics (Eqs. 3 to 12), using the computer program Statistica ${ }^{\oplus}$, version 7.0, through non-linear regression, by the Quasi-Newton method (Statsoft, 2007).

- Newton - Lewis (1921):

$$
\mathrm{MR}=\exp (-\mathrm{kt})
$$

- Page - Page (1949):

$$
\mathrm{MR}=\exp \left(-\mathrm{kt}^{\mathrm{n}}\right)
$$

- Henderson \& Pabis - Henderson \& Pabis (1961):

$$
\mathrm{MR}=\mathrm{a} \exp (-\mathrm{kt})
$$

- Two-Term Exponential - Sharaf-Eldeen et al. (1980):

$$
\mathrm{MR}=\mathrm{a} \exp (-\mathrm{kt})+(1-\mathrm{a}) \exp (-\mathrm{kat})
$$

- Thompson - Thompson et al. (1968):

$$
\mathrm{MR}=\exp \left[-\mathrm{a}\left(\mathrm{a}^{2}+4 \mathrm{bt}\right)^{0.5}\right] / 2 \mathrm{~b}
$$


- Logarithmic - Yagcioglu et al. (1999):

$$
\mathrm{MR}=\mathrm{a} \exp (-\mathrm{kt})+\mathrm{c}
$$

- Approximation of Diffusion - Sharaf-Elden et al. (1980):

$$
\mathrm{MR}=\mathrm{a} \exp (-\mathrm{kt})+(1-\mathrm{a}) \exp (-\mathrm{kbt})
$$

- Modified Henderson \& Pabis - Karathanos (1999):

$$
\mathrm{MR}=\mathrm{a} \exp (-\mathrm{kt})+\mathrm{b} \exp (-\mathrm{kt})+\mathrm{c} \exp (-\mathrm{kt})
$$

- Two Terms - Henderson (1974):

$$
M R=a \exp \left(-\mathrm{k}_{0} \mathrm{t}\right)+\mathrm{b} \exp \left(-\mathrm{k}_{1} \mathrm{t}\right)
$$

- Midilli - Midilli et al. (2002):

$$
\mathrm{MR}=\mathrm{a} \exp \left(-\mathrm{kt}^{\mathrm{n}}\right)+\mathrm{bt}
$$

where:

MR - moisture content ratio, dimensionless;

a, b, c, k, $\mathrm{k}_{0}, \mathrm{k}_{1}, \mathrm{n}$ - coefficients of the models; and,

$\mathrm{t}$ - drying time, min.

The criteria for the fit of the mathematical models to the experimental data were the coefficient of determination $\left(\mathrm{R}^{2}\right)$ (Eq. 13), mean squared deviation (MSD) (Eq. 14), mean relative error (P) (Eq. 15), mean estimated error (SE) (Eq. 16) and the chi-square $\left(\chi^{2}\right)$ (Eq. 17) (Costa et al., 2016; Haas et al., 2017; Rabha et al., 2017).

$$
\begin{aligned}
& \mathrm{R}^{2}=\frac{\sum_{\mathrm{i}=1}^{\mathrm{N}}\left[\left(\mathrm{MR}_{\text {exp }, \mathrm{i}}-\overline{\mathrm{MR}}_{\text {exp }, \mathrm{i}}\right)\left(\mathrm{MR}_{\text {pred }, \mathrm{i}}-\overline{\mathrm{MR}}_{\text {pred }, \mathrm{i}}\right)\right]^{2}}{\sum_{\mathrm{i}=1}^{\mathrm{N}}\left(\mathrm{MR}_{\text {exp }, \mathrm{i}}-\overline{\mathrm{MR}}_{\text {exp }, \mathrm{i}}\right)^{2} \sum_{\mathrm{i}=1}^{\mathrm{N}}\left(\mathrm{MR}_{\text {pred }, \mathrm{i}}-\overline{\mathrm{MR}}_{\text {pred }, \mathrm{i}}\right)^{2}} \\
& \mathrm{MSD}=\left[\frac{1}{\mathrm{~N}} \sum_{\mathrm{i}=1}^{\mathrm{N}}\left(\mathrm{MR}_{\text {pred,i }}-\mathrm{MR}_{\text {exp, } \mathrm{i}}\right)^{2}\right]^{\frac{1}{2}} \\
& \mathrm{P}=\frac{100}{\mathrm{~N}} \sum_{\mathrm{i}=1}^{\mathrm{N}} \frac{\left|\mathrm{MR}_{\text {exp }, \mathrm{i}}-\mathrm{MR}_{\text {pred }, \mathrm{i}}\right|}{\mathrm{MR}_{\text {exp }, \mathrm{i}}} \\
& \mathrm{SE}=\left[\frac{1}{\mathrm{~N}-\mathrm{n}} \sum_{\mathrm{i}=1}^{\mathrm{N}}\left(\mathrm{MR}_{\text {exp }, \mathrm{i}}-\mathrm{MR}_{\text {pred }, \mathrm{i}}\right)^{2}\right]^{\frac{1}{2}} \\
& \chi^{2}=\frac{1}{\mathrm{~N}-\mathrm{n}} \sum_{\mathrm{i}=1}^{\mathrm{N}}\left(\mathrm{MR}_{\mathrm{exp}, \mathrm{i}}-\mathrm{MR}_{\text {pred, } \mathrm{i}}\right)^{2}
\end{aligned}
$$

where:

$\mathrm{MR}_{\exp }$ - experimental moisture content ratio;

$\overline{\mathrm{MR}}_{\text {exp,i }}^{\exp }$ - average experimental moisture content ratio;

$\mathrm{MR}_{\text {pred }}$ - moisture content ratio predicted by the model;

$\overline{\mathrm{MR}}_{\text {pred,i }}$ - average moisture content ratio predicted by the model;
$\mathrm{N}$ - number of observations; and,

$\mathrm{n}$ - number of coefficients of the model.

The geometric shape of the samples was assumed to be that of a cube (parallelepiped with equal sides), and the analytical solution of the second Fick's law for this geometry, considering internal diffusive mass flow equal to the external convective flow in the vicinity of the samples (convective boundary condition) (Eq. 18) (Silva et al., 2014b), was fitted to the experimental data of drying kinetics for determining the effective diffusion coefficients $\left(\mathrm{D}_{\mathrm{ef}}\right)$ and convective mass transfer coefficients $\left(\mathrm{h}_{\mathrm{w}}\right)$, using $16 \times 16 \times 16$ terms of the analytical solution referring to the three summations of Eq. (18), employing the program Convective Adsorption Desorption, version 3.2 (Silva \& Silva, 2018).

$$
\begin{aligned}
\bar{M}(\mathrm{t})= & \mathrm{M}_{\mathrm{eq}}+\left(\mathrm{M}_{0}-\mathrm{M}_{\mathrm{eq}}\right) \sum_{\mathrm{n}=1}^{\infty} \sum_{\mathrm{m}=1}^{\infty} \sum_{\mathrm{k}=1}^{\infty} \mathrm{B}_{\mathrm{n}} \mathrm{B}_{\mathrm{m}} \mathrm{B}_{\mathrm{k}} . \\
& \cdot \exp \left[-\left(\frac{\mu_{\mathrm{n}^{2}}}{\left(\mathrm{~L}_{1} / 2\right)^{2}}+\frac{\mu_{\mathrm{m}^{2}}}{\left(\mathrm{~L}_{2} / 2\right)^{2}} \frac{\mu_{\mathrm{k}^{2}}}{\left(\mathrm{~L}_{3} / 2\right)^{2}}\right) \mathrm{D}_{\mathrm{ef}} \mathrm{t}\right]
\end{aligned}
$$

where:

$\bar{M}(t)$ - average moisture content at time t, d.b.;

$\mathrm{M}_{\mathrm{eq}}$ - equilibrium moisture content, d.b.;

$\mathrm{M}_{0}$ - initial moisture content, d.b.;

$\mu_{n}, \mu_{m}$ and $\mu_{k}$ - roots of the characteristic equation, obtained through Eq. 19;

$\mathrm{B}_{\mathrm{n}}, \mathrm{B}_{\mathrm{m}}$ and $\mathrm{B}_{\mathrm{k}}$ - solution parameters calculated according to Eq. 21;

$\mathrm{D}_{\text {ef }}$ - effective diffusion coefficient, $\mathrm{m}^{2} \mathrm{~s}^{-1}$;

$\mathrm{L}_{1}, \mathrm{~L}_{2}$ and $\mathrm{L}_{3}$ - length, height and thickness, respectively, $\mathrm{m}$; and,

$\mathrm{t} \quad$ - time, $\mathrm{s}$.

$$
\cot \mu_{\mathrm{j}}=\frac{\mu_{\mathrm{j}}}{\mathrm{Bi}}
$$

With $\mathrm{j}$ equal to the indices $\mathrm{n}, \mathrm{m}$ and $\mathrm{k}$. The parameter $\mathrm{Bi}$ is the mass transfer Biot number and is given by Eq. 20:

$$
\mathrm{Bi}=\frac{\mathrm{h}_{\mathrm{w}} \mathrm{L}}{\mathrm{D}_{\mathrm{ef}}}
$$

where:

$h_{w} \quad$ - convective mass transfer coefficient, $\mathrm{m} \mathrm{s}^{-1}$;

L - characteristics length, $\mathrm{m}$; and,

$\mathrm{D}_{\text {ef }}$ - effective diffusion coefficient, $\mathrm{m}^{2} \mathrm{~s}^{-1}$.

$$
B_{j}=\frac{2 B i^{2}}{\mu_{j^{2}}\left(B i^{2}+B i+\mu_{j^{2}}\right)}
$$

where $\mathrm{j}$ is equal to the indices $n, m$ and $k$.

\section{Results AND Discussion}

In the drying curves of the osmotically dehydrated papaya cubes (Figures $1 \mathrm{~A}$ to D), it can be observed that, as the 
concentration and temperature of the solution increase, the drying curves (Figures 1C and D) become less distant from one another, denoting less relative influence of drying temperature on water removal dynamics. Although the increase in concentration and temperature of the solution causes a greater water removal during the OD process (Germer et al., 2011; Souraki et al., 2014), due to the elevation of the concentration gradient of soluble solids between the fruit and the solution, it also results in the incorporation of solute in the sample (GarciaNoguera et al., 2010; Mendes et al., 2013), which could lead to a higher resistance to heat and mass transfers during convective drying, resulting in lower drying effectiveness. Fernandes et al. (2008) demonstrated, in experiments with pineapple, that OD in solutions with high concentrations ( $>35^{\circ} \mathrm{Brix}$ ) results in high gain of solids by the samples, which may cause a reduction in the water removal rate during convective drying.

The drying rates (Figures $2 \mathrm{~A}$ to $\mathrm{D}$ ), for the same concentration of the solution, in general, were higher in samples subjected to solutions at lower temperatures, ranging from 0.85 to $1.64 \mathrm{~kg} \mathrm{~kg}^{-1} \mathrm{~h}^{-1}$ for the samples subjected to OD pre-treatment of $50{ }^{\circ} \mathrm{Brix} / 60^{\circ} \mathrm{C}$ and $50{ }^{\circ} \mathrm{Brix} / 50{ }^{\circ} \mathrm{C}$, respectively, dried at $50{ }^{\circ} \mathrm{C}$.

The values of the drying rates changed over time, gradually increasing to the maximum value and then decreasing

A.

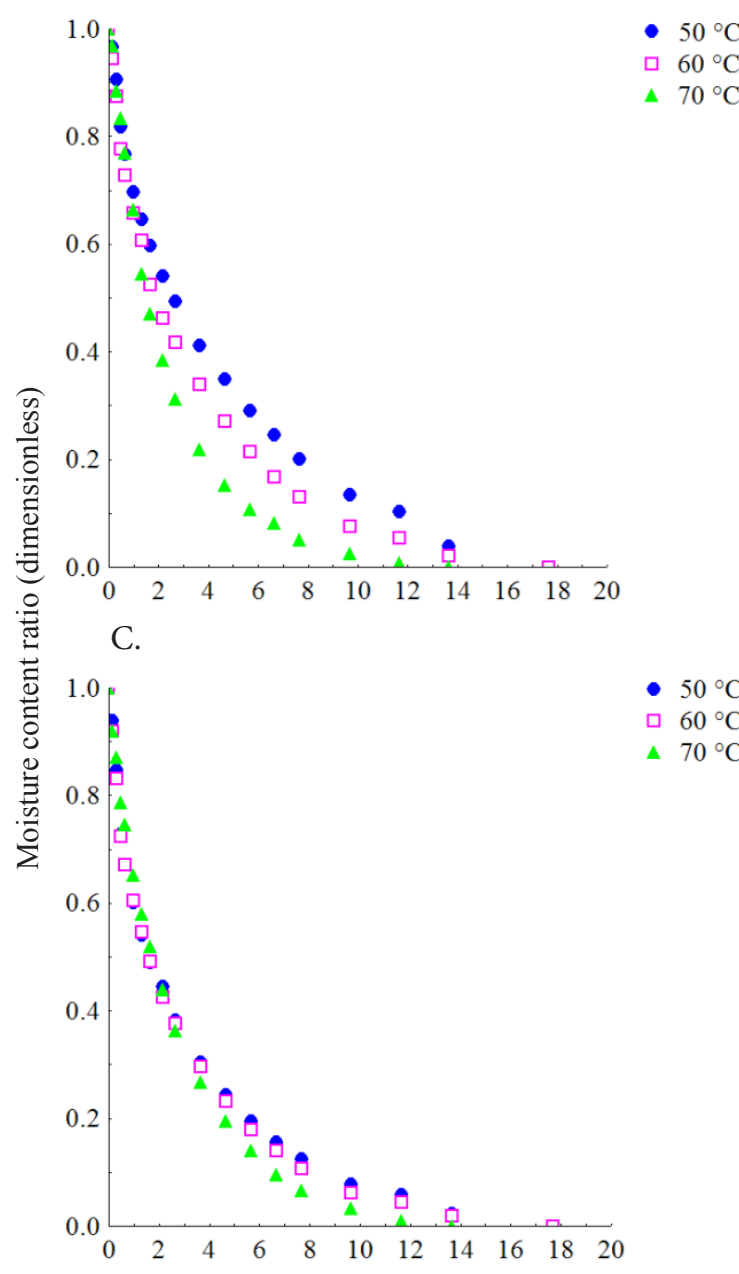

rapidly. This occurs because, at the beginning of the drying, liquid diffusion is the main mechanism of water transport and, as the drying progresses, vapor diffusion becomes the dominant mode, so the drying rate increases. However, with the continuity of the process, the samples become unsaturated with moisture, the vapor diffusion decreases and, consequently, the drying rate also decreases (Chen et al., 2017). In addition, drying occurred mainly in the falling rate period, and no constant rate period was observed (Figures $2 \mathrm{~A}$ to D), indicating that the internal resistance to water movement is greater than the rate of removal from the sample surface (Pilatti et al., 2016). Similar behavior was observed by Kaushal \& Sharma (2016) during convective drying at different temperatures $\left(50-70{ }^{\circ} \mathrm{C}\right)$ of osmo-dehydrated jackfruit pulp.

The indices of the models fitted to the experimental data of drying kinetics of the samples (Table 1), at different temperatures, demonstrate that the Two Terms model had the highest values of the coefficients of determination $\left(\mathrm{R}^{2}\right)$ (0.997-0.999) and the lowest mean squared deviations (MSD) (0.008-0.014), mean relative error (P) (1.306-6.039\%), mean estimated error (SE) (0.009-0.017) and chi-square $\left(\chi^{2}\right)(1.0 \mathrm{x}$ $\left.10^{-4}-3.0 \times 10^{-4}\right)$, so it better represents the drying process of the samples under the studied conditions. However, it should be pointed out that the models of Page, Approximation of

B.

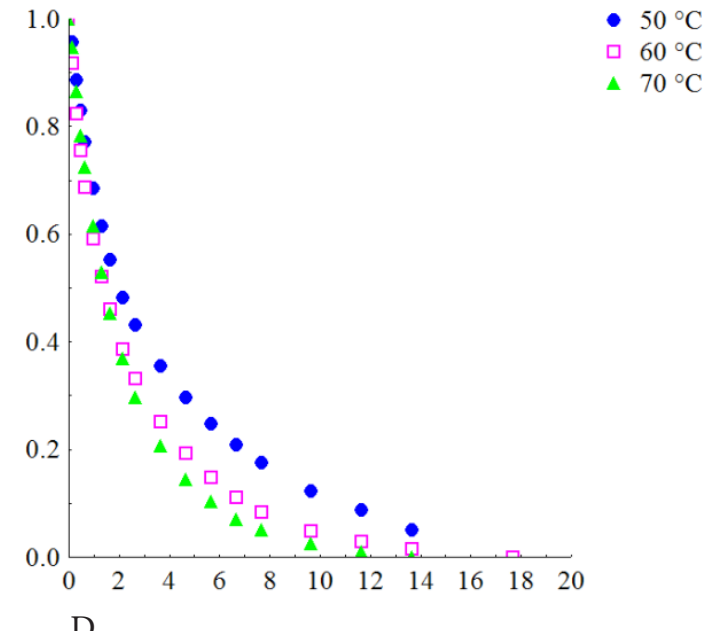

D.
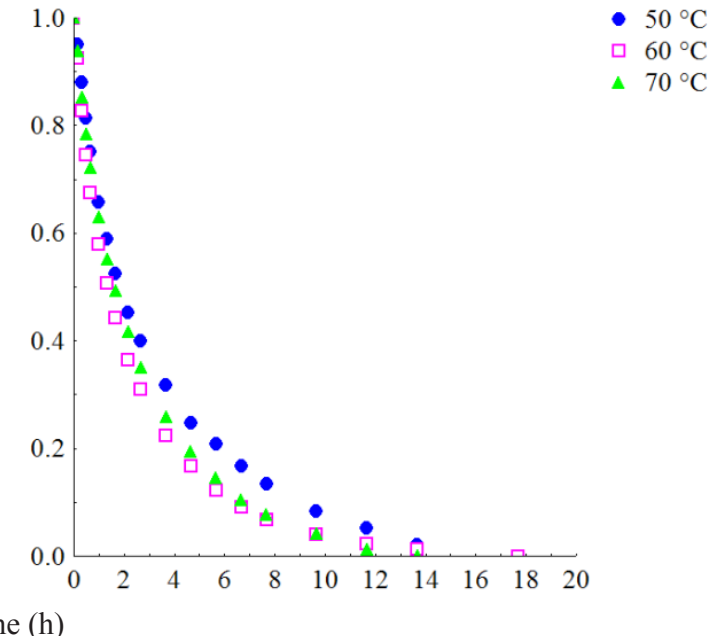

Drying time (h)

Figure 1. Drying curves of papaya cubes subjected to osmotic dehydration at: (A) $40^{\circ} \mathrm{Brix} / 50^{\circ} \mathrm{C}$; (B) $40{ }^{\circ} \mathrm{Brix} / 60{ }^{\circ} \mathrm{C}$; (C) $50{ }^{\circ} \mathrm{Brix} / 50^{\circ} \mathrm{C}$; and (D) $50{ }^{\circ} \mathrm{Brix} / 60^{\circ} \mathrm{C}$ followed by convective drying at temperatures of 50,60 and $70{ }^{\circ} \mathrm{C}$ 
A.

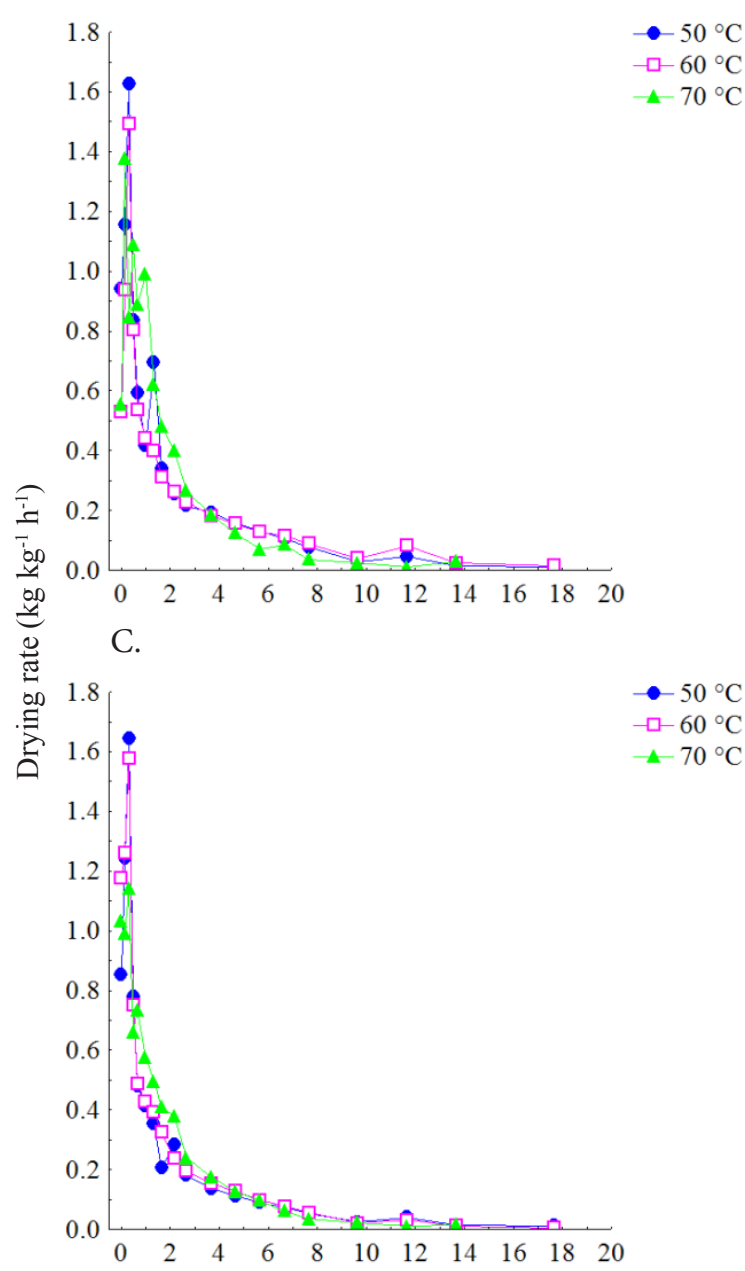

B.

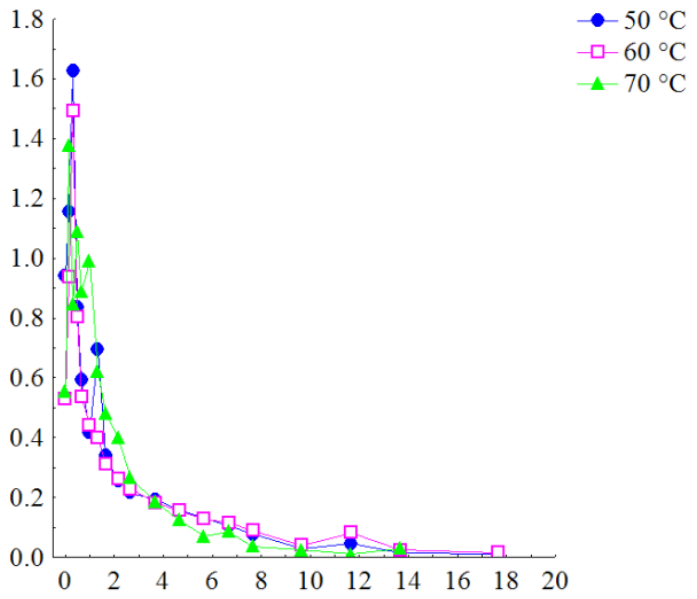

D.

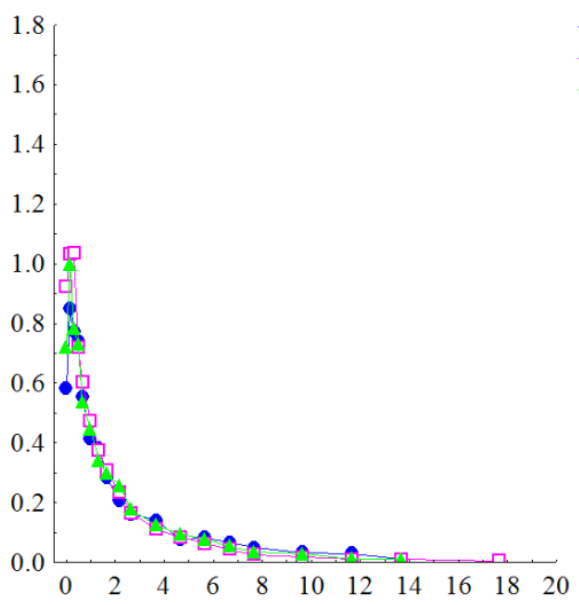

$-50^{\circ} \mathrm{C}$

$-60^{\circ} \mathrm{C}$

$\triangle 70{ }^{\circ} \mathrm{C}$

Drying time $(\mathrm{h})$

Figure 2. Drying rates of papaya cubes subjected to osmotic dehydration at: (A) $40{ }^{\circ} \mathrm{Brix} / 50{ }^{\circ} \mathrm{C}$; (B) $40{ }^{\circ} \mathrm{Brix} / 60{ }^{\circ} \mathrm{C}$; $(\mathrm{C})$ $50{ }^{\circ} \mathrm{Brix} / 50^{\circ} \mathrm{C}$; and (D) $50{ }^{\circ} \mathrm{Brix} / 60^{\circ} \mathrm{C}$ followed by convective drying at temperatures of 50,60 and $70{ }^{\circ} \mathrm{C}$

Table 1. Values of the coefficient of determination $\left(\mathrm{R}^{2}\right)$, mean squared deviation (MSD), mean relative error (P), mean estimated error (SE) and chi-square $\left(\chi^{2}\right)$ of the models fitted to the experimental drying data of papaya cubes subjected to osmotic dehydration $(\mathrm{OD})$

\begin{tabular}{|c|c|c|c|c|c|c|}
\hline \multirow{2}{*}{ Model } & \multirow{2}{*}{ OD } & \multicolumn{5}{|c|}{ Temperature } \\
\hline & & $\mathbf{R}^{2}$ & MSD & $\mathbf{P}(\%)$ & SE & $x^{2}\left(x 10^{-4}\right)$ \\
\hline & & & & $50^{\circ} \mathrm{C}$ & & \\
\hline \multirow{4}{*}{ Newton } & $40^{\circ} \mathrm{Brix} / 50^{\circ} \mathrm{C}$ & 0.976 & 0.047 & 13.536 & 0.049 & 23.0 \\
\hline & $40^{\circ} \mathrm{Brix} / 60^{\circ} \mathrm{C}$ & 0.978 & 0.046 & 19.320 & 0.048 & 23.0 \\
\hline & $50^{\circ} \mathrm{Brix} / 50^{\circ} \mathrm{C}$ & 0.964 & 0.058 & 26.586 & 0.060 & 36.0 \\
\hline & $50^{\circ} \mathrm{Brix} / 60^{\circ} \mathrm{C}$ & 0.985 & 0.038 & 19.136 & 0.040 & 16.0 \\
\hline \multirow{4}{*}{ Page } & $40^{\circ} \mathrm{Brix} / 50^{\circ} \mathrm{C}$ & 0.995 & 0.021 & 8.199 & 0.023 & 5.0 \\
\hline & $40^{\circ} \mathrm{Brix} / 60^{\circ} \mathrm{C}$ & 0.996 & 0.018 & 4.622 & 0.020 & 4.0 \\
\hline & $50^{\circ} \mathrm{Brix} / 50^{\circ} \mathrm{C}$ & 0.995 & 0.021 & 7.488 & 0.022 & 5.0 \\
\hline & $50^{\circ} \mathrm{Brix} / 60^{\circ} \mathrm{C}$ & 0.997 & 0.016 & 6.684 & 0.017 & 3.0 \\
\hline \multirow{4}{*}{ Henderson \& Pabis } & $40^{\circ} \mathrm{Brix} / 50^{\circ} \mathrm{C}$ & 0.986 & 0.036 & 9.661 & 0.039 & 15.0 \\
\hline & $40^{\circ} \mathrm{Brix} / 60^{\circ} \mathrm{C}$ & 0.984 & 0.039 & 15.061 & 0.041 & 17.0 \\
\hline & $50^{\circ} \mathrm{Brix} / 50^{\circ} \mathrm{C}$ & 0.977 & 0.046 & 18.852 & 0.049 & 24.0 \\
\hline & $50^{\circ} \mathrm{Brix} / 60^{\circ} \mathrm{C}$ & 0.989 & 0.033 & 15.009 & 0.035 & 13.0 \\
\hline \multirow{4}{*}{$\begin{array}{l}\text { Two-Term } \\
\text { Exponential }\end{array}$} & $40^{\circ} \mathrm{Brix} / 50^{\circ} \mathrm{C}$ & 0.995 & 0.021 & 7.670 & 0.023 & 5.0 \\
\hline & $40^{\circ} \mathrm{Brix} / 60^{\circ} \mathrm{C}$ & 0.995 & 0.020 & 8.260 & 0.022 & 5.0 \\
\hline & $50^{\circ} \mathrm{Brix} / 50^{\circ} \mathrm{C}$ & 0.988 & 0.033 & 14.118 & 0.035 & 12.0 \\
\hline & $50^{\circ} \mathrm{Brix} / 60^{\circ} \mathrm{C}$ & 0.998 & 0.014 & 6.554 & 0.015 & 2.0 \\
\hline \multirow{4}{*}{ Thompson } & $40^{\circ} \mathrm{Brix} / 50^{\circ} \mathrm{C}$ & 0.993 & 0.025 & 11.478 & 0.027 & 7.0 \\
\hline & $40^{\circ} \mathrm{Brix} / 60^{\circ} \mathrm{C}$ & 0.997 & 0.015 & 4.746 & 0.016 & 3.0 \\
\hline & $50^{\circ} \mathrm{Brix} / 50^{\circ} \mathrm{C}$ & 0.995 & 0.021 & $\begin{array}{c}4.140 \\
13.451\end{array}$ & 0.023 & $\begin{array}{l}5.0 \\
5.0\end{array}$ \\
\hline & $50^{\circ} \mathrm{Brix} / 60^{\circ} \mathrm{C}$ & 0.998 & 0.013 & 10.520 & 0.014 & 2.0 \\
\hline
\end{tabular}


Continuation of Table 1

\begin{tabular}{|c|c|c|c|c|c|c|}
\hline \multirow{2}{*}{ Model } & \multirow{2}{*}{ OD } & \multicolumn{5}{|c|}{ Temperature } \\
\hline & & $\mathbf{R}^{2}$ & MSD & $\mathbf{P}(\%)$ & SE & $\chi^{2}\left(\times 10^{-4}\right)$ \\
\hline \multirow{5}{*}{ Logarithmic } & & & & $50^{\circ} \mathrm{C}$ & & \\
\hline & $40^{\circ} \mathrm{Brix} / 50^{\circ} \mathrm{C}$ & 0.987 & 0.035 & 11.583 & 0.038 & 15.0 \\
\hline & $40^{\circ} \mathrm{Brix} / 60^{\circ} \mathrm{C}$ & 0.990 & 0.031 & 9.606 & 0.034 & 1.2 \\
\hline & $50^{\circ} \mathrm{Brix} / 50^{\circ} \mathrm{C}$ & 0.982 & 0.040 & 18.276 & 0.044 & 20.0 \\
\hline & $50^{\circ}$ Brix $/ 60^{\circ} \mathrm{C}$ & 0.992 & 0.027 & 15.998 & 0.030 & 9.0 \\
\hline \multirow{4}{*}{$\begin{array}{l}\text { Approximation of } \\
\text { Diffusion }\end{array}$} & $40^{\circ} \mathrm{Brix} / 50^{\circ} \mathrm{C}$ & 0.997 & 0.015 & 6.169 & 0.017 & 3.0 \\
\hline & $40^{\circ} \mathrm{Brix} / 60^{\circ} \mathrm{C}$ & 0.998 & 0.010 & 2.540 & 0.012 & 1.0 \\
\hline & $50^{\circ} \mathrm{Brix} / 50^{\circ} \mathrm{C}$ & 0.997 & 0.014 & 4.743 & 0.016 & 3.0 \\
\hline & $50^{\circ} \mathrm{Brix} / 60^{\circ} \mathrm{C}$ & 0.999 & 0.009 & 5.833 & 0.010 & 1.0 \\
\hline \multirow{4}{*}{$\begin{array}{l}\text { Modified } \\
\text { Henderson \& Pabis }\end{array}$} & $40^{\circ} \mathrm{Brix} / 50^{\circ} \mathrm{C}$ & 0.986 & 0.036 & 9.661 & 0.042 & 17.0 \\
\hline & $40^{\circ} \mathrm{Brix} / 60^{\circ} \mathrm{C}$ & 0.984 & 0.039 & 15.059 & 0.044 & 19.0 \\
\hline & $50^{\circ}$ Brix $/ 50^{\circ} \mathrm{C}$ & 0.977 & 0.046 & 18.852 & 0.052 & 27.0 \\
\hline & $50^{\circ} \mathrm{Brix} / 60^{\circ} \mathrm{C}$ & 0.989 & 0.033 & 15.009 & 0.037 & 14.0 \\
\hline \multirow{4}{*}{ Two Terms } & $40^{\circ}$ Brix $/ 50^{\circ} \mathrm{C}$ & 0.997 & 0.014 & 6.039 & 0.017 & 3.0 \\
\hline & $40^{\circ}$ Brix $/ 60^{\circ} \mathrm{C}$ & 0.999 & 0.009 & 1.306 & 0.011 & 1.0 \\
\hline & $50^{\circ} \mathrm{Brix} / 50^{\circ} \mathrm{C}$ & 0.998 & 0.013 & 4.283 & 0.015 & 2.0 \\
\hline & $50^{\circ} \mathrm{Brix} / 60^{\circ} \mathrm{C}$ & 0.999 & 0.008 & 5.392 & 0.009 & 1.0 \\
\hline \multirow{4}{*}{ Midilli } & $40^{\circ} \mathrm{Brix} / 50^{\circ} \mathrm{C}$ & 0.997 & 0.016 & 4.889 & 0.018 & 3.0 \\
\hline & $40^{\circ} \operatorname{Brix} / 60^{\circ} \mathrm{C}$ & 0.997 & 0.016 & 3.869 & 0.018 & 3.0 \\
\hline & $50^{\circ} \mathrm{Brix} / 50^{\circ} \mathrm{C}$ & 0.996 & 0.019 & 5.068 & 0.022 & 5.0 \\
\hline & $50^{\circ}$ Brix $60^{\circ} \mathrm{C}$ & 0.998 & 0.014 & 5.481 & 0.016 & 3.0 \\
\hline \multirow{5}{*}{ Newton } & & & & $60^{\circ} \mathrm{C}$ & & \\
\hline & $40^{\circ} \mathrm{Brix} / 50^{\circ} \mathrm{C}$ & 0.981 & 0.044 & 18.651 & 0.045 & 3.0 \\
\hline & $40^{\circ} \mathrm{Brix} / 60^{\circ} \mathrm{C}$ & 0.984 & 0.040 & 26.478 & 0.041 & 17.0 \\
\hline & $50^{\circ} \operatorname{Brix} / 50^{\circ} \mathrm{C}$ & 0.970 & 0.054 & 26.015 & 0.055 & 30.0 \\
\hline & $50^{\circ} \mathrm{Brix} / 60^{\circ} \mathrm{C}$ & 0.988 & 0.035 & 27.045 & 0.036 & 13.0 \\
\hline \multirow{4}{*}{ Page } & $40^{\circ} \mathrm{Brix} / 50^{\circ} \mathrm{C}$ & 0.997 & 0.016 & 8.094 & 0.017 & 3.0 \\
\hline & $40^{\circ} \mathrm{Brix} / 60^{\circ} \mathrm{C}$ & 0.998 & 0.010 & 4.408 & 0.011 & 1.0 \\
\hline & $50^{\circ} \mathrm{Brix} / 50^{\circ} \mathrm{C}$ & 0.997 & 0.016 & 8.239 & 0.017 & 3.0 \\
\hline & $50^{\circ} \mathrm{Brix} / 60^{\circ} \mathrm{C}$ & 0.999 & 0.006 & 4.720 & 0.012 & 0.4 \\
\hline \multirow{4}{*}{ Henderson \& Pabis } & $40^{\circ} \mathrm{Brix} / 50^{\circ} \mathrm{C}$ & 0.988 & 0.034 & 12.643 & 0.036 & 1.3 \\
\hline & $40^{\circ} \mathrm{Brix} / 60^{\circ} \mathrm{C}$ & 0.989 & 0.032 & 20.612 & 0.034 & 12.0 \\
\hline & $50^{\circ}$ Brix $/ 50^{\circ} \mathrm{C}$ & 0.983 & 0.040 & 17.405 & 0.042 & 18.0 \\
\hline & $50^{\circ} \mathrm{Brix} / 60^{\circ} \mathrm{C}$ & 0.991 & 0.029 & 22.856 & 0.031 & 10.0 \\
\hline & $40^{\circ} \mathrm{Brix} / 50^{\circ} \mathrm{C}$ & 0.996 & 0.018 & 6.444 & 0.019 & 4.0 \\
\hline Two-Term & $40^{\circ} \mathrm{Brix} / 60^{\circ} \mathrm{C}$ & 0.997 & 0.015 & 10.980 & 0.016 & 2.0 \\
\hline Exponential & $50^{\circ} \mathrm{Brix} / 50^{\circ} \mathrm{C}$ & 0.992 & 0.027 & 12.461 & 0.029 & 8.0 \\
\hline & $50^{\circ} \mathrm{Brix} / 60^{\circ} \mathrm{C}$ & 0.998 & 0.011 & 11.170 & 0.012 & 31.0 \\
\hline & $40^{\circ} \mathrm{Brix} / 50^{\circ} \mathrm{C}$ & 0.996 & 0.019 & 14.230 & 0.020 & 4.0 \\
\hline & $40^{\circ}$ Brix $/ 60^{\circ} \mathrm{C}$ & 0.999 & 0.009 & 12.484 & 0.010 & 1.0 \\
\hline Inompson & $50^{\circ}$ Brix $/ 50^{\circ} \mathrm{C}$ & 0.996 & 0.019 & 16.449 & 0.021 & 4.0 \\
\hline & $50^{\circ}$ Brix $/ 60^{\circ} \mathrm{C}$ & 0.999 & 0.008 & 8.103 & 0.009 & 1.0 \\
\hline & $40^{\circ} \mathrm{Brix} / 50^{\circ} \mathrm{C}$ & 0.990 & 0.031 & 13.519 & 0.034 & 12.0 \\
\hline & $40^{\circ}$ Brix $/ 60^{\circ} \mathrm{C}$ & 0.993 & 0.026 & 21.517 & 0.029 & 8.0 \\
\hline Logarıthmıc & $50^{\circ} \mathrm{Brix} / 50^{\circ} \mathrm{C}$ & 0.986 & 0.035 & 17.578 & 0.039 & 1.5 \\
\hline & $50^{\circ} \mathrm{Brix} / 60^{\circ} \mathrm{C}$ & 0.994 & 0.023 & 20.213 & 0.026 & 7.0 \\
\hline & $40^{\circ} \mathrm{Brix} / 50^{\circ} \mathrm{C}$ & 0.998 & 0.012 & 5.500 & 0.013 & 2.0 \\
\hline Approximation of & $40^{\circ} \mathrm{Brix} / 60^{\circ} \mathrm{C}$ & 0.999 & 0.003 & 2.196 & 0.004 & 10.0 \\
\hline Diffusion & $50^{\circ} \mathrm{Brix} / 50^{\circ} \mathrm{C}$ & 0.998 & 0.010 & 3.716 & 0.011 & 1.0 \\
\hline & $50^{\circ} \mathrm{Brix} / 60^{\circ} \mathrm{C}$ & 0.999 & 0.006 & 1.702 & 0.007 & 0.5 \\
\hline & $40^{\circ}$ Brix $/ 50^{\circ} \mathrm{C}$ & 0.988 & 0.034 & 12.647 & 0.039 & 15.0 \\
\hline Modified & $40^{\circ} \mathrm{Brix} / 60^{\circ} \mathrm{C}$ & 0.989 & 0.032 & 20.613 & 0.036 & 1.3 \\
\hline Henderson \& Pabis & $50^{\circ}$ Brix $/ 50^{\circ} \mathrm{C}$ & 0.983 & 0.040 & 17.407 & 0.045 & 20.0 \\
\hline & $50^{\circ} \mathrm{Brix} / 60^{\circ} \mathrm{C}$ & 0.991 & 0.029 & 22.856 & 0.033 & 11.0 \\
\hline & $40^{\circ} \mathrm{Brix} / 50^{\circ} \mathrm{C}$ & 0.998 & 0.011 & 5.369 & 0.013 & 2.0 \\
\hline & $40^{\circ} \mathrm{Brix} / 60^{\circ} \mathrm{C}$ & 0.999 & 0.003 & 1.942 & 0.004 & 0.1 \\
\hline Iwo lerms & $50^{\circ} \operatorname{Brix} / 50^{\circ} \mathrm{C}$ & 0.999 & 0.010 & 3.635 & 0.011 & 1.0 \\
\hline & $50^{\circ} \mathrm{Brix} / 60^{\circ} \mathrm{C}$ & 0.999 & 0.005 & 2.038 & 0.006 & 0.1 \\
\hline & $40^{\circ} \mathrm{Brix} / 50^{\circ} \mathrm{C}$ & 0.998 & 0.014 & 4.634 & 0.016 & 3.0 \\
\hline & $40^{\circ} \mathrm{Brix} / 60^{\circ} \mathrm{C}$ & 0.999 & 0.009 & 3.234 & 0.011 & 1.0 \\
\hline TVIIIIIIII & $50^{\circ} \operatorname{Brix} / 50^{\circ} \mathrm{C}$ & 0.997 & 0.014 & 3.934 & 0.016 & 3.0 \\
\hline & $50^{\circ} \mathrm{Brix} / 60^{\circ} \mathrm{C}$ & 0.999 & 0.010 & 2.899 & 0.012 & 1.0 \\
\hline & & & & $70^{\circ} \mathrm{C}$ & & \\
\hline & $40^{\circ} \mathrm{Brix} / 50^{\circ} \mathrm{C}$ & 0.997 & 0.016 & 7.957 & 0.017 & 3.0 \\
\hline & $40^{\circ}$ Brix $/ 60^{\circ} \mathrm{C}$ & 0.997 & 0.016 & 14.011 & 0.017 & 3.0 \\
\hline Newton & $50^{\circ}$ Brix $/ 50^{\circ} \mathrm{C}$ & 0.996 & 0.019 & 8.749 & 0.020 & 4.0 \\
\hline & $50^{\circ} \mathrm{Brix} / 60^{\circ} \mathrm{C}$ & 0.993 & 0.027 & 14.204 & 0.028 & 8.0 \\
\hline
\end{tabular}

Continues on the next page 
Continuation of Table 1

\begin{tabular}{|c|c|c|c|c|c|c|}
\hline \multirow{2}{*}{ Model } & \multirow{2}{*}{ OD } & \multicolumn{5}{|c|}{ Temperature } \\
\hline & & $\mathrm{R}^{2}$ & MSD & $\mathrm{P}(\%)$ & SE & $\chi^{2}\left(x 10^{-4}\right)$ \\
\hline \multirow{5}{*}{ Page } & & & & $70^{\circ} \mathrm{C}$ & & \\
\hline & $40^{\circ} \mathrm{Brix} / 50^{\circ} \mathrm{C}$ & 0.997 & 0.016 & 8.646 & 0.017 & 3.0 \\
\hline & $40^{\circ} \mathrm{Brix} / 60^{\circ} \mathrm{C}$ & 0.998 & 0.011 & 5.748 & 0.012 & 1.0 \\
\hline & $50^{\circ} \mathrm{Brix} / 50^{\circ} \mathrm{C}$ & 0.999 & 0.007 & 10.705 & 0.008 & 1.0 \\
\hline & $50^{\circ} \mathrm{Brix} / 60^{\circ} \mathrm{C}$ & 0.999 & 0.010 & 7.352 & 0.011 & 1.0 \\
\hline \multirow{4}{*}{ Henderson \& Pabis } & $40^{\circ} \mathrm{Brix} / 50^{\circ} \mathrm{C}$ & 0.998 & 0.015 & 9.638 & 0.016 & 3.0 \\
\hline & $40^{\circ} \mathrm{Brix} / 60^{\circ} \mathrm{C}$ & 0.998 & 0.015 & 12.944 & 0.016 & 3.0 \\
\hline & $50^{\circ} \mathrm{Brix} / 50^{\circ} \mathrm{C}$ & 0.998 & 0.014 & 7.317 & 0.015 & 2.0 \\
\hline & $50^{\circ} \mathrm{Brix} / 60^{\circ} \mathrm{C}$ & 0.995 & 0.023 & 10.214 & 0.024 & 6.0 \\
\hline \multirow{4}{*}{$\begin{array}{l}\text { Two-Term } \\
\text { Exponential }\end{array}$} & $40^{\circ} \mathrm{Brix} / 50^{\circ} \mathrm{C}$ & 0.997 & 0.017 & 7.664 & 0.018 & 3.0 \\
\hline & $40^{\circ} \mathrm{Brix} / 60^{\circ} \mathrm{C}$ & 0.998 & 0.015 & 12.490 & 0.016 & 3.0 \\
\hline & $50^{\circ} \mathrm{Brix} / 50^{\circ} \mathrm{C}$ & 0.999 & 0.008 & 6.161 & 0.009 & 1.0 \\
\hline & $50^{\circ} \mathrm{Brix} / 60^{\circ} \mathrm{C}$ & 0.999 & 0.006 & 6.212 & 0.007 & 1.0 \\
\hline \multirow{4}{*}{ Thompson } & $40^{\circ}$ Brix $/ 50^{\circ} \mathrm{C}$ & 0.997 & 0.015 & 7.137 & 0.017 & 3.0 \\
\hline & $40^{\circ} \mathrm{Brix} / 60^{\circ} \mathrm{C}$ & 0.999 & 0.008 & 6.037 & 0.009 & 1.0 \\
\hline & $50^{\circ} \mathrm{Brix} / 50^{\circ} \mathrm{C}$ & 0.999 & 0.011 & 16.197 & 0.012 & 2.0 \\
\hline & $50^{\circ} \mathrm{Brix} / 60^{\circ} \mathrm{C}$ & 0.998 & 0.010 & 13.652 & 0.012 & 1.0 \\
\hline \multirow{4}{*}{ Logarithmic } & $40^{\circ} \mathrm{Brix} / 50^{\circ} \mathrm{C}$ & 0.998 & 0.013 & 14.501 & 0.015 & 2.0 \\
\hline & $40^{\circ} \mathrm{Brix} / 60^{\circ} \mathrm{C}$ & 0.998 & 0.012 & 15.520 & 0.014 & 2.0 \\
\hline & $50^{\circ} \mathrm{Brix} / 50^{\circ} \mathrm{C}$ & 0.998 & 0.013 & 10.994 & 0.015 & 2.0 \\
\hline & $50^{\circ}$ Brix $/ 60^{\circ} \mathrm{C}$ & 0.996 & 0.019 & 16.281 & 0.022 & 5.0 \\
\hline \multirow{4}{*}{$\begin{array}{l}\text { Approximation of } \\
\text { Diffusion }\end{array}$} & $40^{\circ}$ Brix $/ 50^{\circ} \mathrm{C}$ & 0.998 & 0.015 & 8.900 & 0.017 & 4.0 \\
\hline & $40^{\circ} \mathrm{Brix} / 60^{\circ} \mathrm{C}$ & 0.999 & 0.007 & 5.498 & 0.008 & 1.0 \\
\hline & $50^{\circ} \mathrm{Brix} / 50^{\circ} \mathrm{C}$ & 0.999 & 0.005 & 7.349 & 0.006 & 0.1 \\
\hline & $50^{\circ} \mathrm{Brix} / 60^{\circ} \mathrm{C}$ & 0.999 & 0.006 & 6.037 & 0.007 & 1.0 \\
\hline \multirow{4}{*}{$\begin{array}{l}\text { Modified } \\
\text { Henderson \& Pabis }\end{array}$} & $40^{\circ} \mathrm{Brix} / 50^{\circ} \mathrm{C}$ & 0.998 & 0.015 & 9.638 & 0.017 & 3.0 \\
\hline & $40^{\circ} \mathrm{Brix} / 60^{\circ} \mathrm{C}$ & 0.998 & 0.015 & 12.944 & 0.017 & 3.0 \\
\hline & $50^{\circ} \mathrm{Brix} / 50^{\circ} \mathrm{C}$ & 0.998 & 0.014 & 7.317 & 0.016 & 3.0 \\
\hline & $50^{\circ} \mathrm{Brix} / 60^{\circ} \mathrm{C}$ & 0.995 & 0.022 & 10.217 & 0.026 & 7.0 \\
\hline \multirow{4}{*}{ Two Terms } & $40^{\circ} \mathrm{Brix} / 50^{\circ} \mathrm{C}$ & 0.998 & 0.011 & 8.802 & 0.013 & 2.0 \\
\hline & $40^{\circ} \mathrm{Brix} / 60^{\circ} \mathrm{C}$ & 0.999 & 0.006 & 4.541 & 0.007 & 1.0 \\
\hline & $50^{\circ} \mathrm{Brix} / 50^{\circ} \mathrm{C}$ & 0.999 & 0.006 & 7.319 & 0.007 & 0.1 \\
\hline & $50^{\circ} \mathrm{Brix} / 60^{\circ} \mathrm{C}$ & 0.999 & 0.006 & 5.760 & 0.007 & 0.1 \\
\hline \multirow{4}{*}{ Midilli } & $40^{\circ} \mathrm{Brix} / 50^{\circ} \mathrm{C}$ & 0.998 & 0.014 & 10.848 & 0.016 & 2.0 \\
\hline & $40^{\circ} \mathrm{Brix} / 60^{\circ} \mathrm{C}$ & 0.999 & 0.009 & 6.712 & 0.011 & 1.0 \\
\hline & $50{ }^{\circ} \mathrm{Brix} / 50^{\circ} \mathrm{C}$ & 0.999 & 0.005 & 2.141 & 0.006 & 0.1 \\
\hline & $50^{\circ} \mathrm{Brix} / 60^{\circ} \mathrm{C}$ & 0.999 & 0.008 & 3.954 & 0.010 & 1.0 \\
\hline
\end{tabular}

Diffusion and Midilli, also had high $\mathrm{R}^{2}$ values, above 0.995, and low MSD, P, SE and $\chi^{2}$, below 0.021, 8.199\%, 0.023 and 5.0 $\mathrm{x} 10^{-4}$, respectively, indicating their adequacy to represent the drying kinetics of osmotically pre-dehydrated papaya cubes.

Table 2 presents the effective diffusion coefficients $\left(D_{\text {ef }}\right)$ and convective mass transfer coefficients $\left(h_{w}\right)$ obtained for the drying of the samples, subjected to the temperatures of 50 , 60 and $70{ }^{\circ} \mathrm{C}$. The increase in convective drying temperature $\left(50-70{ }^{\circ} \mathrm{C}\right)$ causes the increment in $\mathrm{D}_{\text {ef }}$ values. In addition, it was observed that samples subjected to the solutions with the same temperature, but with higher sucrose concentration, in particular for the drying temperature of $70{ }^{\circ} \mathrm{C}$, offer greater resistance to external mass transfer, which in turn may be related to the reduction of $h_{w}$. This behavior may be associated with increased concentration of soluble solids during the OD, on the surface of the sample (Rodríguez et al., 2015; Sangeeta

Table 2. Effective diffusion coefficients $\left(\mathrm{D}_{\text {ef }}\right)$ and convective mass transfer coefficients $\left(\mathrm{h}_{\mathrm{w}}\right)$ obtained in convective drying, at temperatures (Temp) of 50,60 and $70^{\circ} \mathrm{C}$, of osmotically pre-dehydrated (OD) papaya cubes

\begin{tabular}{|c|c|c|c|c|c|c|}
\hline OD & $\begin{array}{l}\text { Temp } \\
\left({ }^{\circ} \mathrm{C}\right)\end{array}$ & $\begin{array}{c}\mathbf{h}_{\mathrm{w}} \\
\left(\mathrm{m} \mathrm{s}^{-1}\right)\end{array}$ & $\begin{array}{c}D_{\text {ef }} \\
\left(m^{2} s^{-1}\right)\end{array}$ & Bi & $\mathbf{R}^{2}$ & $\chi^{2}$ \\
\hline \multirow{3}{*}{$40^{\circ} \mathrm{Brix} / 50^{\circ} \mathrm{C}$} & 50 & $5.697 \times 10^{-7}$ & $8.440 \times 10^{-10}$ & 6.750 & 0.996 & $6.571 \times 10^{-3}$ \\
\hline & 60 & $7.411 \times 10^{-7}$ & $1.097 \times 10^{-9}$ & 6.750 & 0.998 & $3.944 \times 10^{-3}$ \\
\hline & 70 & $3.910 \times 10^{-7}$ & $3.910 \times 10^{-6}$ & 0.001 & 0.998 & $4.885 \times 10^{-3}$ \\
\hline \multirow{3}{*}{$40^{\circ}$ Brix $/ 60^{\circ} \mathrm{C}$} & 50 & $6.553 \times 10^{-7}$ & $9.709 \times 10^{-10}$ & 6.750 & 0.997 & $5.746 \times 10^{-3}$ \\
\hline & 60 & $9.742 \times 10^{-7}$ & $1.443 \times 10^{-9}$ & 6.750 & 0.999 & $2.050 \times 10^{-3}$ \\
\hline & 70 & $5.845 \times 10^{-7}$ & $4.031 \times 10^{-9}$ & 1.450 & 0.998 & $2.850 \times 10^{-3}$ \\
\hline \multirow{3}{*}{$50^{\circ} \mathrm{Brix} / 50^{\circ} \mathrm{C}$} & 50 & $1.201 \times 10^{-6}$ & $9.611 \times 10^{-10}$ & 12.500 & 0.996 & $7.161 \times 10^{-3}$ \\
\hline & 60 & $1.138 \times 10^{-6}$ & $1.059 \times 10^{-9}$ & 10.750 & 0.998 & $3.577 \times 10^{-3}$ \\
\hline & 70 & $5.622 \times 10^{-7}$ & $2.444 \times 10^{-9}$ & 2.300 & 0.999 & $6.693 \times 10^{-4}$ \\
\hline \multirow{3}{*}{$50^{\circ} \mathrm{Brix} / 60^{\circ} \mathrm{C}$} & 50 & $6.661 \times 10^{-7}$ & $1.268 \times 10^{-9}$ & 5.250 & 0.997 & $4.561 \times 10^{-4}$ \\
\hline & 60 & $9.273 \times 10^{-7}$ & $1.766 \times 10^{-10}$ & 5.250 & 0.998 & $2.811 \times 10^{-4}$ \\
\hline & 70 & $6.680 \times 10^{-7}$ & $1.979 \times 10^{-9}$ & 3.375 & 0.999 & $1.605 \times 10^{-4}$ \\
\hline
\end{tabular}

Bi - Biot number; $\chi^{2}$ - Chi-square 
\& Hathan, 2016; Goula et al., 2017), capable of forming, at high temperatures $\left(\geq 70{ }^{\circ} \mathrm{C}\right)$, a dense and poorly permeable layer, increasing the resistance to heat transfer to the samples and establishing an additional barrier to the water exit from its interior (Munhoz et al., 2014; Corrêa et al., 2017). Similar results have been reported in strawberry (Garcia-Noguera et al., 2010) and plum (Dehghannya et al., 2016).

It is observed that the Biot number ( $\mathrm{Bi}$ ) was within the range from 0.001 to 12.500 (Table 2), which, according to Kaya et al. (2010), is indicative of the existence of internal and external resistances to water transfer, being considered the most realistic case in practical applications. It should be pointed out that $\mathrm{Bi}$ tended to decrease with the elevation in the drying temperature, especially at $70{ }^{\circ} \mathrm{C}$, indicating that there is a higher resistance to mass flow on the surface of the samples (Silva et al., 2013).

The solution of the Fick's second law equation (Eq. 18), for all OD treatments, considering convective boundary condition, showed, even in Biot number $<<1\left(\mathrm{Bi}=0.001 ; \mathrm{R}^{2}=0.998 ; \chi^{2}\right.$ $\left.=4.885 \times 10^{-3}\right)$, adequate fit to the experimental data of drying kinetics of the samples $\left(\mathrm{R}^{2}>0.996\right.$ and $\left.\chi^{2}<7.161 \times 10^{-3}\right)$ (Table 2 ), which ensures the physical representativeness of the values of $\mathrm{D}_{\text {ef }}$ and $\mathrm{h}_{\mathrm{w}}$.

\section{Conclusions}

1. Among the fitted mathematical models, the Two Terms model was selected as the most adequate for drying kinetics of osmo-dehydrated papaya cubes.

2. The effective diffusivity in the samples increased with the increase of air temperature, whereas the convective mass transfer coefficient showed a less defined trend.

\section{ACKnowledgments}

The authors thank the Conselho Nacional de Desenvolvimento Científico e Tecnológico (CNPq) for the financial support.

\section{Literature Cited}

Chen, Q.; Bi, J.; Chen, R.; Liu, X.; Wu, X.; Zhou, M. Comparative study on drying characteristic, moisture diffusivity, and some physical and nutritional attributes of blanched carrot slices. Journal of Food Process and Preservation, v.41, p.1-8, 2017. https://doi. org/10.1111/jfpp.13201

Corrêa, J. L. G.; Rasia, M. C.; Mulet, A. J.; Cárcel, A. Influence of ultrasound application on both the osmotic pretreatment and subsequent convective drying of pineapple (Ananas comosus). Innovative Food Science and Emerging Technologies, v.41, p.284291, 2017. https://doi.org/10.1016/j.ifset.2017.04.002

Costa, C. F.; Corrêa, P. C.; Vanegas, J .D. B.; Baptestini, F. M.; Campos, R. C.; Fernandes, L. S. Mathematical modeling and determination of thermodynamic properties of jabuticaba peel during the drying process. Revista Brasileira de Engenharia Agrícola e Ambiental, v.20, p.576-580, 2016. https://doi.org/10.1590/1807-1929/ agriambi.v20n6p576-580
Dehghannya, J.; Gorbani, R.; Ghanbarzadeh, B. Shrinkage of mirabelle plum during hot air drying as influenced by ultrasound-assisted osmotic dehydration. International Journal of Food Properties, v.19, p.1093-1103, 2016. https://doi.org/10.1080/10942912.201 5.1055362

Dermesonlouoglou, E.; Chalkia, A.; Taoukis, P. Application of osmotic dehydration to improve the quality of dried goji berry. Journal of Food Engineering, v.232, p.36-43, 2018. https://doi.org/10.1016/j. jfoodeng.2018.03.012

FAOSTAT - Food and Agriculture Organization of the United Nations Statistics Division. Available on: <http://faostat3.fao.org/ browse/Q/QC/E>. Access on: Apr. 2018.

Fernandes, F. A. N.; Linhares Jr., F. E.; Rodrigues, S. Ultrasound as pre-treatment for drying of pineapples. Ultrasonics Sonochemistry, v.15, p.1049-1054, 2008. https://doi.org/10.1016/j. ultsonch.2008.03.009

Galaz, P.; Valdenegro, M.; Ramírez, C.; Nuñez, H.; Almonacid, S.; Simpson, R. Effect of drum drying temperature on drying kinetic and polyphenol contents in pomegranate peel. Journal of Food Engineering, v.208, p.19-27, 2017. https://doi.org/10.1016/j. jfoodeng.2017.04.002

Garcia-Noguera, J.; Oliveira, F. I. P.; Gallão, M. I.; Weller, C. L.; Rodrigues, S.; Fernandes, F. A. N. Ultrasound-assisted osmotic dehydration of strawberries: effect of pre-treatment time and ultrasonic frequency. Drying Technology, v.28, p.294-303, 2010. https://doi.org/10.1080/07373930903530402

Gava, A. J.; Silva, C. A. B. da.; Frias, J. R. G. Tecnologia de alimentos: Princípios e aplicações. Nobel, São Paulo, 2008.

Germer, S. P. M.; Queiroz, M. R. de.; Aguirre, J. M.; Berbari, S. A. G.; Anjos, V. D. Desidratação osmótica de pêssegos em função da temperatura e concentração do xarope de sacarose. Revista Brasileira de Engenharia Agrícola e Ambiental, v.15, p.161-169, 2011. https://doi.org/10.1590/S1415-43662011000200008

Goula, A. M.; Kokolaki, M.; Daftsiou, E. Use of ultrasound for osmotic dehydration. The case of potatoes. Food and bioproducts processing, v.105, p.157-170, 2017. https://doi.org/10.1016/j. fbp.2017.07.008

Haas, I. C. da S.; Toaldo, I. M.; Müller, C. M. O.; Bordignon-Luiz, M. T. Modeling of drying kinetics of the non-pomace residue of red grape (V. labrusca L.) juices: Effect on the microstructure and bioactive anthocyanins. Journal of food process engineering, v.40, p.1-11, 2017. https://doi.org/10.1111/jfpe.12568

Henderson, S. M. Progress in developing the thin layer drying equation. Transactions of the American Society of Agricultural Engineers, v.17, p.1167-1168, 1974. https://doi.org/10.13031/2013.37052

Henderson, S. M.; Pabis, S. Grain drying theory. II: Temperature effects on drying coefficients. Journal of Agricultural Engineering Research, v.6, p.169-174, 1961.

Lewis, W. K. The rate of drying of solid materials. The Journal of Industrial and Engineering Chemistry, v.13, p.427-432, 1921. https://doi.org/10.1021/ie50137a021

Kandasamy, P.; Varadharaju, N.; Kalemullah, S.; Moitra, R. Production of papaya powder under foam-mat drying using methyl cellulose as foaming agent. Asian Journal of Food and Agro-Industry, v.5, p.374-387, 2012.

Karathanos, V. T. Determination of water content of dried fruits by drying kinetics. Journal of Food Engineering, v.39, p.337-344, 1999. https://doi.org/10.1016/S0260-8774(98)00132-0 
Kaya, A.; Aydın, O.; Dincer, I. Comparison of experimental data with results of some drying models for regularly shaped products. Heat Mass Transfer, v.46, p.555-562, 2010. https://doi.org/10.1007/ s00231-010-0600-z

Kaushal, P.; Sharma, H. K. Osmo-convective dehydration kinetics of jackfruit (Artocarpus heterophyllus). Journal of the Saudi Society of Agricultural Sciences, v.15, p.118-126, 2016. https:// doi.org/10.1016/j.jssas.2014.08.001

Kucuk, H.; Midilli, A.; Kilic, A.; Dincer, I. A review on thin-layer drying-curve equations. Drying Technology, v.32, p.757-773, 2014. https://doi.org/10.1080/07373937.2013.873047

Mendes, G. R. L.; Freitas, C. H. de; Scaglioni, P. T.; Schmidt, C. G.; Furlong, E. B. Condições para desidratação osmótica de laranjas e as propriedades funcionais do produto. Revista Brasileira de Engenharia Agrícola e Ambiental, v.17, p.1210-1216, 2013. https:// doi.org/10.1590/S1415-43662013001100012

Midilli, A.; Kucuk, H.; Yapar, Z. A new model for single layer drying. Drying Technology, v.20, p.1503-1513, 2002. https://doi. org/10.1081/DRT-120005864

Munhoz, C. L.; Sanjinez-Argandoña, E. J.; Campagnolli, R.; Macedo, M. L. R. Drying of the kernel and fresh and osmotically dehydrated bocaiuva pulps. v.36, p.165-170, 2014. https://doi. org/10.4025/16843

Orikasa, T.; Koide, S.; Okamoto, S.; Imaizumi, T.; Muramatsu, Y.; Takeda, J.; Shiina, T.; Tagawa, A. Impacts of hot air and vacuum drying on the quality attributes of kiwifruit slices. Journal of Food Engineering, v.125, p.51-58, 2014. https://doi.org/10.1016/j. jfoodeng.2013.10.027

Özdemira, M. B.; Aktaşa, M.; Şevik, S.; Khanlari, A. Modeling of a convective-infrared kiwifruit drying process. International Journal of Hydrogen Energy, v.42, p.18005-18013, 2017. https:// doi.org/10.1016/j.ijhydene.2017.01.012

Pacheco-Angulo, H.; Herman-Lara, E.; García-Alvarado, M. A.; Ruiz-López, I. I. Mass transfer modeling in osmotic dehydration: Equilibrium characteristics and process dynamics under variable solution concentration and convective boundary. Food and Bioproducts Processing, v.97, p.88-99, 2016. https://doi. org/10.1016/j.fbp.2015.11.002

Page, G. E. Factors influencing the maximum rates of air drying shelled corn in thin layers. West Lafayette: Purdue University, 1949. Thesis Doctoral

Pilatti, D.; Johann, G.; Palú, F.; Silva, E. A. da. Evaluation of a concentrated parameters mathematical model applied to drying of yerba mate leaves with variable mass transfer coefficient. Applied Thermal Engineering, v.105, p.483-489, 2016. https:// doi.org/10.1016/j.applthermaleng.2016.02.139

Prosapio, V.; Norton, I. Influence of osmotic dehydration pretreatment on oven drying and freeze drying performance. LWT - Food Science and Technology, v.80, p.401-408, 2017. https://doi. org/10.1016/j.lwt.2017.03.012

Rabha, D. K.; Muthukumar, P.; Somayaji, C. Experimental investigation of thin layer drying kinetics of ghost chilli pepper (Capsicum chinense Jacq.) dried in a forced convection solar tunnel dryer. Renewable Energy, v.105, p.583-589, 2017. https:// doi.org/10.1016/j.renene.2016.12.091
Rodríguez, M. M.; Rodriguez, A.; Mascheroni, R. H. Color, texture, rehydration ability and phenolic compounds of plums partially osmodehydrated and finish-dried by hot air. Journal of Food Processing and Preservation, v.39, p.2647-2662, 2015. https:// doi.org/10.1111/jfpp.12515

Sangeeta; Hathan, B. S. studies on mass transfer and diffusion coefficients in elephant foot yam (Amorphophallus SPP.) during osmotic dehydration in sodium chloride solution. Journal of Food Processing and Preservation, v.40, p.521-530, 2016. https://doi. org/10.1111/jfpp.12631

Sharaf-Eldeen, Y. I.; Blaisdell, J. L.; Hamdy, M. Y. A model for ear corn drying. Transactions of the American Society of Agricultural Engineers, v.23, p.1261-1265, 1980. https://doi. org/10.13031/2013.34757

Silva, W. P.; Silva, C. M. D. P. S. Convective Adsorption - Desorption, Versão 3.2. (2008 - 2018). Available on: <http://zeus.df.ufcg.edu. br/labfit/Convective.htm>. Access on: Sep. 2018.

Silva, W. P.; Silva, C. M. D. P. S.; Gomes, J. P. Drying description of cylindrical pieces of bananas in different temperatures using diffusion models. Journal of Food Engineering, v.117, p.417-424, 2013. https://doi.org/10.1016/j.jfoodeng.2013.03.030

Silva, W. P.; Silva, C. M. D. P. S.; Aires, J. E. F.; Silva-Junior, A. F. Osmotic dehydration and convective drying of coconut slices: Experimental determination and description using one-dimensional diffusion model. Journal of the Saudi Society of Agricultural Sciences, v.13, p.162-168, 2014a. https://doi. org/10.1016/j.jssas.2013.05.002

Silva, W. P.; Silva, C. M. D. P. S.; Lins, M. A. A.; Gomes, J. P. Osmotic dehydration of pineapple (Ananas comosus) pieces in cubical shape described by diffusion models. LWT - Food Science and Technology, v.55, p.1-8, 2014b. https://doi.org/10.1016/j. lwt.2013.08.016

Souraki, B. A.; Ghavami, M.; Tondro, H. Correction of moisture and sucrose effective diffusivities for shrinkage during osmotic dehydration of apple in sucrose solution. Food and Bioproducts Processing, v.92, p.1-8, 2014. https://doi. org/10.1016/j.fbp.2013.07.002

Statsoft. Statistica for Windows - Computer program manual. Version 7.0 Tulsa: Statsoft Inc., 2007.

Thompson, T. L.; Peart, R. M.; Foster, G. H. Mathematical simulation of corn drying: A new model. Transactions of the American Society of Agricultural Engineers, v.11, p.582-586, 1968. https:// doi.org/10.13031/2013.39473

Tzempelikos, D. A.; Mitrakos, D.; Vouros, A. P.; Bardakas, A. V.; Filios, A. E.; Margaris, D. P. Numerical modeling of heat and mass transfer during convective drying of cylindrical quince slices. Journal of Food Engineering, v.156, p.10-21, 2015. https://doi. org/10.1016/j.jfoodeng.2015.01.017

Yagcioglu, A.; Degirmencioglu, A.; Cagatay, F. Drying characteristics of laurel leaves under different conditions. In: International Congress on Agricultural Mechanization and Energy, 7, 1999, Adana. Proceedings... Adana: Faculty of Agriculture, Cukurova University, 1999, p.565-569. 